\title{
Kennzeichen flexibler, agiler Organisationen und ihre Bedeutung für Führung und Wohlbefinden bei der Arbeit
}

\author{
C. Weber \\ M. Ribbat \\ Bundesanstalt für Arbeitsschutz und Arbeitsmedizin (BAuA), \\ Fachgruppe Arbeitszeit und Organisation, Dortmund
}

(eingegangen am 12.07.2021, angenommen am 12.11.2021)

ABSTRACT / ZUSAMMENFASSUNG

\section{Characteristics of flexible, agile organisations and their significance for leadership and well-being at work}

Introduction: Agility is an organisational concept to increase the adaptability and flexibility of companies and it is widely discussed among researchers and practitioners. However, in many cases, the term agility is not precisely defined. Moreover, it describes very different approaches.

Aims: The aim of this article is to identify essential characteristics and requirements of agile working methods in organisations that have established alternative structures and processes in order to improve adaptability and flexibility.

Method: We conducted and analysed qualitative interviews $(\mathrm{N}=27)$ in five organisations.

Results: We found characteristics of innovative and flexible forms of leadership and organisation in transformed structures and processes, defined values and objectives as an orientation framework and a new understanding of leadership in the companies studied. Transparency and participation were identified as factors that supported agile forms of organisation. The findings were discussed and classified with particular regard to health and well-being. Conclusions: The implementation of agile working requires suitable organisational support for teams, individual employees and leaders in order to be both successful and health-oriented.

Keywords: agility - adaptability - flexibility - leadership

ASU Arbeitsmed Sozialmed Umweltmed 2021; 56: 738-745

\section{Einleitung und Zielstellung}

Im allgemeinen Sprachgebrauch ist der Begriff „Agilität“ ein Modewort und wird häufig eher undifferenziert verwendet. Für die wissenschaftliche Forschung spielt er, im Zuge der Einordnung und Untersuchung von aktuellen, vielfach angewandten Konzepten der Arbeitsorganisation, jedoch eine wichtige Rolle. Bendel (2019) definiert "Agilität" beispielsweise als „die Gewandtheit, Wendigkeit oder Beweglichkeit von Organisationen und Personen bzw. in Strukturen und Prozessen.“ (Bendel 2019, o.S.). Dabei geht es darum, flexibel auf unvorhergesehene Ereignisse und neue Anforderungen zu reagieren und mit Blick aufVeränderungen nicht nur reaktiv, sondern auch proaktiv zu handeln (Bendel 2019). Um wettbewerbsfähig zu bleiben, zielen agile Organisationen nach Walter (2021) auf folgende

\section{Kennzeichen flexibler, agiler Organisationen und ihre Bedeutung für Führung und Wohlbefinden bei der Arbeit}

Einleitung: Agilität ist ein in der Forschung und Praxis viel diskutiertes Organisationskonzept zur Steigerung von Anpassungsfähigkeit und Flexibilität von Unternehmen. Allerdings wird der Begriff Agilität oftmals nicht eindeutig verwendet.

Ziel: Ziel dieses Beitrags ist daher die Identifikation von grundlegenden Kennzeichen und Voraussetzungen agiler Arbeitsweisen in Organisationen, die zur Steigerung von Anpassungsfähigkeit und Flexibilität alternative Strukturen und Prozesse etabliert haben.

Methode: Es wurden qualitative Interviews $(\mathrm{N}=27)$ in fünf Organisationen durchgeführt und ausgewertet.

Ergebnisse: Bei den untersuchten Unternehmen waren veränderte Strukturen und Prozesse, definierte Werte und Ziele als Orientierungsrahmen sowie ein gänzlich verändertes Führungsverständnis als Kennzeichen innovativer und flexibler Führungs- und Organisationsformen zu finden. Transparenz und Partizipation konnten als Faktoren identifiziert werden, die agile Organisationsformen unterstützen. Die Ergebnisse werden mit besonderem Blick auf Gesundheit und Wohlbefinden diskutiert und eingeordnet.

Schlussfolgerungen: Für die erfolgreiche und gesundheitsorientierte Umsetzung bedarf es auch bei agiler Arbeit geeigneter organisationaler Unterstützung für die Teams, die einzelnen Beschäftigten sowie die Führungskräfte. Schlüsselwörter: Agilität - Anpassungsfähigkeit - Flexibilität - Führungskräfte

Aspekte ab: schnelle und effiziente Produktion hochwertiger und kostenreduzierter Produkte, Kundenzufriedenheit, Beschäftigtenzufriedenheit sowie eine erhöhte Geschwindigkeit der Entwicklung und Markteinführung neuer Produkte sowie die Eliminierung von Fehlern und nichtwertschöpfender Prozesse.

Die Anwendungsfelder agiler Arbeitsweisen finden sich in den verschiedenen Branchen über die unterschiedlichen Unternehmensbereiche hinweg von Entwicklung, Produktion bis hin zurVerwaltung (Porschen-Hueck et al. 2020). Unternehmen setzen agile Arbeitsweisen für eine verbesserte Produkteinführungszeit, eine gesteigerte Produktqualität, eine Risikoreduktion im Projekt sowie eine Optimierung der Teammoral ein (Komus et al. 2020). Auf der anderen Seite werden mit agilen Arbeitsweisen ein erhöhter Zeitund Leistungsdruck und eine interessierte Selbstgefährdung, zum 
Beispiel durch die Erstellung von Produktinkrementen in festgelegten Arbeitszyklen, in Zusammenhang gebracht, die sich negativ auf die Gesundheit und das Wohlbefinden der Beschäftigten bei der Arbeit auswirken können (Becke 2020). Der Zusammenhang agiler Arbeitsweisen und Gesundheit ist bisher wenig untersucht.

Es ist wichtig klarzustellen, dass es keine eindeutige Definition von Agilität gibt (Walter 2021).Vielmehr existiert eine Sammlung unterschiedlichster Ansätze mit verschiedenen Schwerpunkten wie die agile Organisationsentwicklung, die Zusammenarbeit im Team, selbstorganisiertes Lernen, Führung etc.

Ausgehend von dieser Uneindeutigkeit und Mehrdimensionalität des Agilitätsbegriffs stellt sich die Frage nach übergreifenden Kennzeichen undVoraussetzungen umgesetzter agiler Arbeitsweisen in Organisationen, die wir explorativ in diesem qualitativ empirischen Beitrag beantworten möchten. Einen besonderen Fokus richten wir dabei auf Führung in agilen Organisationen und diskutieren anhand von Forschungsergebnissen aus anderen Studien zudem den Zusammenhang unserer Befunde mit Gesundheit und Wohlbefinden bei der Arbeit.

\section{Theoretische Einordnung}

Eine eindeutige theoretische Einbettung des Agilitätsbegriffs ist nur schwer möglich. Der Agilitätsbegriff basiert vielmehr auf einer „Sammlung von Elementen verschiedener organisationstheoretischer Ansätze und enthält eineVielzahl organisationaler Konzepte" (Förster u. Wendler 2012, S. 1). Darüber hinaus lassen sich agile Grundgedanken bereits in Theorien und Konzepten finden, die noch keinen direkten Bezug zur agilen Arbeitsweise hergestellt haben (Förster u. Wendler 2012).

Der Grundgedanke eines iterativen Vorgehens und einer Weiterentwicklung durch Prozessveränderungen ist beispielsweise bereits in den 1950er Jahren durch William Demings und seinen „DemingKreis" entstanden (Schermuly u. Koch 2019).

Die Auseinandersetzung mit der Anpassungsfähigkeit von Systemen als wichtige Grundlage des Agilitätsgedankens findet sich zum einen in den naturwissenschaftlichen Systemtheorien von Varela und Maturana (1987), zum anderen aber auch in den Theorien zu Kennzeichen und Prozessen sozialer Systeme (Luhmann 2000). So wird in der Organisationsliteratur insbesondere "systemischen“ Organisationen eine hohe Anpassungsfähigkeit zugeschrieben (Laloux 2014). Im Verständnis Parsons $(1951,2012)$ und Luhmanns (2000) verfügen Organisationen als soziale Systeme über eine organisationsspezifische Eigenlogik. Diese ermöglicht es ihnen, selbstorganisiert aufVeränderungen von außen zu reagieren. Organisationen sind demnach zwar physisch offene Systeme, sie lassen sich aufgrund ihrer stukturellen Eigenlogik aber nur sehr bedingt von außen steuern. Somit lässt sich die Bedeutung von Selbstorganisation als ein Element von Agilität bereits in den sozialen Systemtheorien finden.

Die im Agilitätsbegiff enthaltene Fokussierung auf den Menschen und seine Zufriedenheit lässt sich auf den Human-Relations-Ansatz und seine Weiterentwicklung zum Human-Ressourcen-Ansatz zurückführen. Der Human-Relations-Ansatz, der in den 1930er Jahren als Kritik an der Tayloristischen Arbeitsweise entstanden war, fokussiert neben Effizienz auch auf die Zufriedenheit der Beschäftigten und ihre Beziehungen untereinander (Nerdinger et al. 2014). Der Human-Ressourcen-Ansatz geht dabei einen Schritt weiter und betrachtet auch Strukturen und Prozesse als veränderbar in Hinblick auf

\section{KERNAUSSAGEN}

- Agilität als Organisationskonzept findet in vielen Unternehmen Anwendung, um die Anpassungsfähigkeit und Flexibilität der Unternehmen zu steigern. Der Begriff „Agilität“ wird nicht immer eindeutig verwendet und kann sich beispielsweise auf Arbeitsweisen und -methoden, eine Haltung oder auf die Beweglichkeit eines gesamten organisationalen Systems beziehen.

- Veränderte Strukturen und Prozesse, ein klarer Orientierungsrahmen z.B. durch definierte Werte und Ziele sowie ein gänzlich verändertes Führungsverständnis waren Kennzeichen innovativer und flexibler Führungs- und Organisationsformen bei den in diesem Beitrag untersuchten Unternehmen.

- Agile Arbeitsweisen bieten einige Chancen für Organisationen und Beschäftigte, wie beispielsweise mehr Anpassungsfähigkeit, Flexibilität oder Autonomie.

- Flexibilisierung und Eigenverantwortung können allerdings auch mit Risiken einhergehen wie beispielsweise Entgrenzung der Arbeit, Arbeitsintensivierung oder interessierte Selbstgefährdung.

- Transparenz und Partizipation können dabei unterstützen, die Potenziale von agilen Arbeitsweisen zu nutzen

die Mitarbeitendenmotivation, ihre Beziehung und die Berücksichtigung menschlicher Bedürfnisse. Als einer der wichtigsten Ansätze für Agilität wurden Konzepte wie Selbstorganisation, kontinuierliches Lernen, Partizipation, Zufriedenheit und Selbstkontrolle unter diesem Ansatz diskutiert (Förster u. Wendler 2012).

Die Verknüpfung der dargestellten Konzepte der verschiedenen Organisationstheorien mit dem Agilitätsbegriff führen Förster und Wendler (2012) auf den weit rezipierten Lehigh-Report des Iacocca Institute aus dem Jahre 1991 zurück. Dieser Bericht analysiert den Rückgang der Wettbewerbsfähigkeit der US-Industrie in den 1970er Jahren und sieht Empfehlungen vor, die unter dem Begriff der Agilität zusammengefasst wurden und als Auslöser für weitere Forschung zum Thema Agilität gelten. Erste Agilitätsdefinitionen der 1990er Jahre beschränkten sich zunächst auf die schnelle und flexible Reaktion aufVeränderungen aus dem Außen. Spätere Agilitätsdefinitionen sind weitaus komplexer und umfassender. Yusuf et al. (1999) definieren Agilität beispielsweise als "the successful exploration of competitive bases (speed, flexibility, innovation proactivity, quality and profitability) through the integration of reconfigurable resources and best practices in a knowledge-rich environment to provide customer-driven products and services in a fast changing market environment" (S. 37). Aus einem Vergleich verschiedener Definitionen organisationaler Agilität identifiziert Walter (2021) drei zentrale Charakteristika. Organisationale Agilität gilt als Antwort auf kontinuierliche und unvorhersehbare Veränderungen. Sie fokussiert auf Geschwindigkeit und Anpassungsfähigkeit und zielt auf eine erhöhte Wettbewerbsfähigkeit ab.

Der Einfluss der verschiedenen Theoriestränge auf den Agilitätsbegriff erklärt seine Mehrdimensionalität. Aktuelle Ansätze dazu, wie eine Transformation zu einer adaptiven, flexiblen und lernenden Organisation gelingen kann, sind vielfältig und mehrdimensional (Appelbaum et al. 2017). Agilitätskonzepte lassen sich dabei unterschiedlichen Ebenen zuordnen. Meredith und Francis (2000) adressieren beispielsweise insgesamt 16 voneinander abhängige Dimensionen von Agilität, die sich den vier Ebenen Strategien, Prozesse, Menschen und Verbindungen zuordenen lassen. Haider 
et al. (2021) identifizieren in ihrem Review die Ebenen der organisationalen Agilität, Business Agilität, operationalen Agilität und strategischen Agilität.

Neben der Forschung zur Anwendung von Agilität auf unterschiedlichen Ebenen, nimmt die Agilitätsforschung auch aktuelle Fragen wie zum Beispiel die Einführung von Industrie-4.0-Technologie, Nachhaltigkeitsbemühungen und Umweltziele sowie die Resilienz in Krisenzeiten wie die der Covid-19-Pandemie in den Blick (VazquezBrustelo et al. 2007; Mrugalska u. Ahmed 2021). Die Anpassung des Agilitätsbegriffs an aktuelle Entwicklungen bewerten Förster und Wendler (2012) als eine Stärke des Begriffs, der beschrieben wird als „Konglomerat von Elementen verschiedener Theorien und Konzepte, die fortlaufend um neue Ansätze erweitert wurden“ (S. 13). Als Schwäche stellen sie dieser Stärke die fehlende Einheitlichkeit einer Agilitätsdefinition gegenüber.

Aufgrund der fehlenden einheilichen Agilitätsdefinition im Forschungsdiskurs sowie der uneinheitlichen Verwendung des Agilitätsbegriffs in den untersuchten Organisationen wird der vorliegenden Forschungsarbeit in Anlehnung an Appelbaum (2017) ein weit gefasster Agilitätsbegriff zugrunde gelegt. Agilität erfasst zum einen konkrete Arbeitstechniken mit dem Fokus auf Flexibilität, Partizipation und Selbstorganisation, bezieht sich aber auch auf die Anpassungsfähigkeit des gesamten organisationalen Systems inklusive Organisationsstrukturen, Kultur und Führungsverständnis. Organisationale Agilität beschreibt dabei die Fähigkeit, in einen dynamischen, vom kontinuierlichen, oft unvorhersebaren Wandel bestimmten Umfeld zu funktionieren und wettbewerbsfähig zu bleiben (Appelbaum et al. 2017).

Der Zusammenhang von agilen Arbeitsweisen sowie Gesundheit bei der Arbeit ist noch nicht umfassend erforscht. Einzelne Studien liegen aber vor. Koch und Schermuly (2021) untersuchen beispielsweise, inwieweit Beschäftigte in agilen Projektteams besser mit den durch die Covid-19-Pandemie hervorgerufenen Anforderungen zurecht kommen. Sie sehen in der agilen Arbeisweise eine Jobressource. Tuomivaara et al. (2017) ermitteln eine gleichmäßigere Verteilung der Arbeitsbelastung, aber gleichzeitig auch mehr Belastungen durch Zeitdruck in agilen Teams. Aus den Studien von Venkatesh et al. (2020) und Laanti (2013) geht hervor, dass Erschöpfung in agilen Teams im Zusammenhang mit Zeit- und Leistungsdruck, einem hohen Maß an sozialen Interaktionen, kontinuierlicherVeränderung und Rollenunklarheit steht. Entscheidend für das Belastungsempfinden sind Ressourcen wie organisatorische Fertigkeiten, Rollenklarheit und Empowerment (Venkatesh et al. 2020; Laanti 2013). Laanti (2013) schlussfolgert, dass es letzten Endes auf die Führung und die Gestaltung der agilen Arbeitsweise ankommt. Im Rahmen dieses Beitrags sollen die identifizierten Kennzeichen agiler und flexibler Orgnisationen daher auch mit Blick auf Gesundheit und Wohlbefinden reflektiert werden.

\section{Methodisches Vorgehen}

Der Forschungsfrage nach grundlegenden Kennzeichen undVoraussetzungen agiler Arbeitsweisen entsprechend wurde für diesen Beitrag mit dem Fallstudiendesign (Yin 2018) ein qualitativ-explorativer Forschungsansatz gewählt. Ziel der Untersuchung war es nicht, aus der Theorie abgeleitete Hypothesen zu überprüfen, sondern dem Forschungsgegenstand offen zu begegnen und die Forschungshypothe- sen aus dem Datenmaterial zu entwickeln und wiederum an ihm zu überprüfen (Brüsemeister 2008; Lamnek u. Krell 2016).

Das Kriterium für die Fallauswahl vor dem Hintergrund der Zielstellung (s. oben) war ein hoher Grad an flexiblen und innovativen Organisations- und Führungsformen. Konkret bedeutete dies, dass solche Unternehmen ins Sample aufgenommen wurden, die nach eigenen Angaben in ihrer Organisation grundlegendeVeränderungen von Strukturen und Prozessen in Hinblick auf Flexibilitätsoptionen, die Selbstorganisation von Teams und Beschäftigten und auf die $\mathrm{Zu}$ friedenheit der Beschäftigten durchgeführt hatten.

Die Suche nach Organisationen, die das oben genannte Kriterium erfüllten, erfolgte in erster Linie über das Internet, Social Media Plattformen wie LinkedIn und Xing sowie unter den teilnehmenden Organisationen in anderen Forschungsprojekten der Forschungseinrichtung (Bundesanstalt für Arbeitsschutz und Arbeitsmedizin). In einem telefonischen Erstgespräch wurde grundlegend geklärt, welche Veränderungen im Unternehmen durchgeführt wurden, um die Passung zur Forschungsfrage sicher zu stellen.

Zur Kontrastierung agiler Organisationscharakteristika wurden fünf Organisationen (vgl. Tabelle 1) ausgewählt. Die Motive und Ausgangssituationen in den einzelnen Organisationen für die durchgeführten Veränderungen waren sehr unterschiedliche. Dazu zählten organisatorische Notwendigkeiten, wie hohe Abwesenheiten der Geschäftsführung, wirtschaftliche Zwänge zur Effizienzsteigerung durch Beschäftigtenmotivation oder eine grundsätzliche Haltung in Bezug auf Vereinbarkeitsfragen. Diese Motive und die historisch unterschiedliche Ausgangssituation standen nicht im Fokus unserer Analyse, so dass die Analyseperspektive in erster Linie auf die Umsetzung von Flexibilitätsoptionen, erweiterte Möglichkeiten der Selbstorganisation sowie die Erfahrungen damit gerichtet war. $\mathrm{Zu}$ dem wiesen die fünf Organisationen strukturelle Unterschiede hinsichtlich ihrer Unternehmensgröße und Branchenzugehörigkeit auf.

$\Rightarrow$ Tabelle 1 gibt einen Überblick zur Stichprobe der fünf verschiedenen Organisationen, in denen die Interviews geführt wurden. Sowohl die Branchenzugehörigkeit als auch die Anzahl der Beschäftigten variierte erheblich. Über alle Organisationen hinweg wurden insgesamt 27 Interviews geführt. Zu den interviewten Personen zählten Mitglieder der Geschäftsführung (GF), Führungskräfte (FK) und Mitarbeitende (MA) oder alternativ Mitglieder des Betriebsrats (BR). Dieses Vorgehen sollte es ermöglichen, Diskrepanzen zwischen den Perspektiven sichtbar zu machen (Flick et al. 2015).

Zur Datenerhebung wurden teilstandardisierte, leitfadengestützte und problemzentrierte Interviews, entweder einzeln oder mit zwei Interviewpartnerinnen/-partnern im Tandem, von 60- bis 90-minütiger Dauer durchgeführt. Teilstandardisierte Interviews (vs. Vollstandardisierung) bieten für die Interviewenden die Möglichkeit, situationsbedingte Variationen hinsichtlich der exakten Frageformulierung, der Nachfragestrategie oder auch der Reihenfolge der Fragen zu realisieren (Döring u. Bortz 2016). Der Interviewleitfaden wurde systematisch auf Basis der "SPSS-Methode“ von Helfferich (2011) entwickelt und sollte die Interviewten zu Erzählungen anregen, die möglichst viele der interessierenden Aspekte beinhalteten. So zum Beispiel eine Frage zum Thema Führung „Wie wirken sich die beschriebenen Veränderungen auf die Führung aus?“

Diese Problemzentriertheit berücksichtigte, dass die Forschenden über ein theoretisch-wissenschaftliches Vorverständnis zum Beispiel 
Tabelle 1: Stichprobe/Organisationsübersicht Table 1: Random sample/organisation overview

\begin{tabular}{|l|c|c|c|c|c|}
\hline \multirow{2}{*}{$\begin{array}{l}\text { Organisation } \\
\text { Branche }\end{array}$} & A & B & C & D & E \\
\cline { 2 - 6 } Beschäftigte & Beratung & Öffentliche Daseinsvorsorge & Produktentwicklung und Marketing & Soziale Arbeit & Dienstleistung \\
\cline { 2 - 6 } & ca. 20 & ca. 40.000 & ca. 130 & ca. 400 & GF: 1 \\
N=27 & GF: 1 & GF: 1 & GF: 1 & FK: 5 & GF: 1 \\
& FK: 0 & FK: 1 & FK: 4 & MA: 1 & FR: 4 \\
MA: 1 & BR: 2 & MA: 2 & BR: & \\
\hline
\end{tabular}

GF = Geschäftsführung, FK = Führungskraft, $M A=$ Mitarbeitende, $B R=$ Betriebsratsmitglied

zum Thema Führung verfügen, das sie zur Eingrenzung entscheidender Aspekte des Problembereichs einsetzten. Die Interviewten hatten innerhalb dieser Eingrenzung durch entsprechende Erzählstimuli die Möglichkeit, ihre eigene soziale Realität darzustellen. Durch den Auswertungsprozess und die Erhebung der Daten wurde es den Forschenden ermöglicht, ihre wissenschaftlichen Konzeptionen anzupassen und zu systematisieren (Lamnek u. Krell 2016).

Für die Datenauswertung wurden alle Interviews in Anlehnung an Dresing und Pehl (2018) transkribiert und anschließend gemäß der Grounded Theory nach Glaser und Strauss ausgewertet, da diese insbesondere für ein induktives, exploratives Vorgehen geeignet ist. Der Analyseprozess umfasste dabei verschiedene Stufen des Kodierens und ermöglichte es, die Komplexität des agilen Arbeitens abzubilden. Auf Basis der Daten konnten Hypothesen entwickelt werden, die iterativ an dem Datenmaterial überprüft wurden. Dieser zirkuläre Forschungsprozess beinhaltete einen kontinuierlichen Wechsel zwischen Datenerhebung, -auswertung und Ergebnisreflexion (Strauss u. Corbin 1996). Die Auswertung der Daten erfolgte unter Anwendung der Software MAXQDA (VERBI Software 2020).

\section{Ergebnisse}

Unsere Ergebnisse zeigen, dass die untersuchten Unternehmen auf verschiedenen Ebenen ansetzten, um anpassungsfähig und flexibel agieren zu können. Auf struktureller Ebene wurde durch dezentrale Lösungen die Selbstorganisation der Teams gestärkt. Auf Prozessebene wurden unter anderem durch den Einsatz neuer Technologien erweiterte Flexibilitätsoptionen für die Beschäftigten in Bezug auf den Arbeitsort und die Arbeitszeit geschaffen. Dabei wurde in einigen der Unternehmen besonderer Wert darauf gelegt, dass die Teams selbstbestimmt ihre neue Arbeitsweise (Verantwortlichkeiten, Kombination von Präsenz- und ortsflexibler Arbeit, Definition von Erreichbarkeiten etc.) definieren, ausprobieren und anpassen konnten. Bei der teamspezifischen Festlegung und Ausgestaltung der Arbeitsweisen half ein Orientierungsrahmen, basierend auf transparenten Organisationswerten, einer geteilten Vision, konkreten Zielen und der kontinuierlichen Kommunikation zur Zielerreichung. Die verschiedenen Elemente dieses Orientierungsrahmens wurden partizipativ entwickelt und in klaren und kontinuierlichen Kommunikationsmechanismen hinterfragt, angepasst und weiterentwickelt.

Voraussetzung für diese Veränderungen auf den unterschiedlichen Ebenen waren soziale Beziehungen, die geprägt waren durch Vertrauen und ein Führungsverständnis, in dem die Führungskraft nicht in ers- ter Linie steuert, sondern unterstützt und als Austauschpartnerin/partner für die Beschäftigten zurVerfügung stand.

\section{Strukturen und Prozesse}

In allen untersuchten Organisationen wurden Veränderungen zur Steigerung der Flexibilitätsgrade vorgenommen, etwa durch die Einführung hoher Zeit- und Ortsflexibilität der Arbeit sowie mehr Selbstorganisation für Teams und einzelne Beschäftigte. Die freie Wahl des Arbeitsortes und flexible Arbeitszeiten ermöglichten den Beschäftigten beispielsweise eine bessere Vereinbarkeit von Berufsund Privatleben. Sie berichteten, dass sie die gewährten Flexibilitätsoptionen als Wertschätzung ihrer Person und Anerkennung ihrer Bedürfnisse wahrnahmen. Zudem wurde in diesem Zusammenhang die Bedeutung von Vertrauen betont. Das Kontrollprinzip durch Führungskräfte trat zugunsten von Vertrauen in die Kompetenzen und Leistungsbereitschaft der Beschäftigten in den Hintergrund und diente als Grundlage für flexibles und selbstorganisiertes Arbeiten. Dazu die Aussage einer Mitarbeiterin:

"Aber trotzdem sind wir quasi in den Aufgaben, die wir machen, ja/ also werden da wenig kontrolliert, oder eigentlich nicht. Ne? Also es wird davon ausgegangen, dass wir unsere Arbeit erledigen, und das auch in bestem Wissen und Gewissen" (F3W02MA:103).

Die selbstorganisierte Arbeitsweise der Beschäftigten als Sachverständige ihrer jeweiligen Aufgabe implizierte nicht nur Flexibilitätsoptionen, sondern auch Anforderungen in Bezug aufVerantwortungsübernahme und eigenständiges Treffen von Entscheidungen.

Ein besonderes Merkmal der untersuchten Organisationen zeigte sich in der Prozessgestaltung, die durch ein hohes Maß an technikunterstützter Transparenz, klaren Kommunikationsstrukturen und Partizipationsmöglichkeiten geprägt war. In einer der untersuchten Organisationen wurde diese technikunterstützte Transparenz beispielsweise durch den Einsatz einer entsprechenden Managementsoftware erzeugt. Durch diese wurde allen Beschäftigten uneingeschränkter Zugriff auf alle relevanten Informationen fachlicher Art, aber auch zu wirtschaftlichen Kennzahlen, Bearbeitungsständen und Zuständigkeiten ermöglicht. Verschiedene technische Optionen wurden zudem für unterschiedliche Kommunikationsformate und -inhalte eingesetzt und strukturierten die Zusammenarbeit über Distanz. So erfolgte in einer Organisation beispielsweise die private, informelle Kommunikation über verschiedene Chatkanäle, während für wöchentliche Teambesprechungen Videokonferenzsys- 
teme eingesetzt wurden und 1-zu-1-Kommunikation häufig über Telefon stattfand.

Partizipationsmöglichkeiten auf Prozessebene wurden in den untersuchten Organisationen auf unterschiedliche Weise realisiert. In einem Unternehmen erfolgte beispielsweise eine systematische regelmäßige Einbindung der einzelnen Teams in den Zielfindungsprozess des Unternehmens über integrierte Bottom-up- und Topdown-Prozesse, die dann als Zielindikatoren für alle transparent digital zugänglich gemacht wurden. Innerhalb dieser Prozesse bestand für die Teams die Möglichkeit, Belastungsgrenzen aufzuzeigen und bei der Festlegung von Zielen zu berücksichtigen. In einem anderen Unternehmen bestand die Möglichkeit der Entwicklung von teamspezifischen Regeln der Zusammenarbeit in speziell dafür vorgesehenen Räumlichkeiten und Unterstützung durch Moderation bis hin zu regelmäßig stattfindenden Workshops.

Wie das unten stehende Zitat einer Führungskraft verdeutlicht, ging es dabei darum, die individuellen Bedürfnisse aller Teammitglieder und die des Teams zur Entwicklung von Lösungsansätzen für die zukünftige Zusammenarbeit in den Vordergrund zu stellen.

„Was BRAUCHT ihr? Und was braucht es dazu, dass es jetzt auch funktioniert? Und damit habe ich auch sehr stark pointiert, die Menschen wirklich EINzubeziehen und nicht mit fertigen Lösungen sozusagen zu kommen" (F401FK:9).

\section{Orientierung durch Werte und Ziele}

Die selbstorganisierte Arbeitsweise war eingebettet in einen Orientierungsrahmen, der für die Beschäftigten eine wichtige Funktion erfüllte. In einem Unternehmen bildete eine stark wertebasierte Vision die Grundlage für diesen Orientierungsrahmen. Konkretisiert wurde die durch die Vision vorgegebene Ausrichtung über konsistente Organisations-, Team- und Individualziele. Die starke Durchdringung der Organisation durch das Wertesystem mit zentralen Aspekten wie Wertschätzung, Beteiligung, Respekt, Vertrauen und Transparenz ermöglichte eine systematische Prozessbeteiligung der Beschäftigten bei der Entwicklung von Zielen auf allen Ebenen. Das Wertesystem erfüllte die Funktion eines „Fixsterns“, an dem alles Handeln ausgerichtet und kontinuierlich überprüft wurde. Systematische Kommunikationsstrukturen zur Überprüfung von Zielen und Handlungsschritten erzeugten ein hohes Commitment und eine Verantwortungsübernahme seitens der Beschäftigten. In anderen Unternehmen dienten die Ziele selbst als Orientierungsfunktion für die Beschäftigten. Die dahinterliegenden Werte wurden dabei nicht in allen Fällen explizit gemacht.

Als wichtige Voraussetzung für die Durchdringung der Werte innerhalb einer Organisation konnte die Haltung der Leitung und deren Authentizität bei der expliziten Kommunikation, aber auch Prüfung und gegebenenfalls Anpassung der entsprechenden Werte, identifiziert werden. Erleichternd wirkte es, wenn die Werteorientierung der Beschäftigten bereits bei der Selektion beziehungsweise Einstellung der Beschäftigten berücksichtigt werden konnte und sich so eine gute Passung der Organisations- und persönlichen Werte ergab. Die auf dem Wertesystem basierten strukturellen Möglichkeiten des Hinterfragens und Neu-Ausrichtens von Zielen und Prozessen zeigten sich zum Beispiel in einer Fehlerkultur, bei der es nicht um die Suche nach Schuldigen, sondern um einen humorvollen Umgang
Tabelle 2: Ergebnisübersicht Tabelle 2: Summary oft he results

\begin{tabular}{|l|l|}
\hline Handlungsebene & Merkmal erhöhter Anpassungsfähigkeit \\
\hline Strukturen & Dezentrale Lösungen \\
\hline Prozesse & Selbstorganisation, Flexibilitätsoptionen \\
\hline Orientierung & Partizipativ entwickelte Werte und Ziele \\
\hline Soziale Beziehungen & Vertrauen, Führungskräfte als Unterstützer \\
\hline
\end{tabular}

mit Fehlern und Widersprüchen ging. So berichteten Beschäftigte einer Organisation in ihren Teamrunden von regelmäßigen Austauschen zu so genannten „Fuck-Ups“, damit eine konstruktive, angstfreie Bearbeitung von Schwierigkeiten stattfinden konnte, indem Unterstützungsmöglichkeiten erkennbar wurden und deren Umsetzung wieder zu einer Stärkung des Teamgefüges beitrug. Zudem wurde betont, welche Lernmöglichkeiten sich aus Fehlern ergeben, wenn die Gründe berücksichtigt wurden:

"Ja, und auch zu sagen, wenn irgendwas nicht funktioniert hat oder wir irgendwas nicht erreicht haben, dass es kein Fail ist, sondern dass es einen Grund dafür gibt. Aus dem man wiederum lernt. Und sagt, okay, entweder wir haben uns zu viel vorgenommen, oder es gab personell irgendwie Schwierigkeiten, oder der Weg war der falsche" (F3W04HR:121).

\section{Führungsverständnis}

Damit die selbstorganisierte Arbeitsweise funktionieren kann, bedarf es neben entsprechenden Strukturen und Prozessen sowie eines starken Orientierungsrahmens außerdem eines neuen Führungsverständnisses, das von der klassischen Rolle der Führungskraft als Macherin/Macher und Entscheiderin/Entscheider mit Wissensvorsprung und Kontrollmandat abrückt. Selbstorganisierte Arbeitsweisen setzen voraus, dass Führungskräfte eigenständige Entscheidungen und Impulse aus dem Team zulassen. Für ein solches Führungsverständnis ist insbesondere die innere Haltung der Führungskräfte von Bedeutung. Die Haltung und das Vertrauen der Führungskräfte in ihre Teams wurden in allen teilnehmenden Organisationen als wichtiges Entwicklungsfeld des Kulturwandels identifiziert.

Eine Führungshaltung, die berücksichtigt, dass jede Person unabhängig von der hierarchischen Position etwas beizutragen hat, erleichtert die Erarbeitung und Umsetzung erfolgreicher Teamlösungen durch Diskussionen und Meinungsäußerung über Hierarchieebenen hinweg. Führungskräfte erfüllen an dieser Stelle eine wichtige Funktion. Sie ermöglichen und unterstützen Transparenz und Partizipation in den Teamprozessen und stehen in Hinblick auf die Umsetzung wertebasierterVisionen in operative Ziele und Prozessschritte ihren Teams als Vorbild voran. In den Organisationen, die großen Wert auf eine solche Haltung bei ihren Führungskräften legten, wurde diese entsprechend bei der Selektion und der Karriereförderung für die Führungskräfte berücksichtigt. Ein solches Führungsverständnis, wie es in einigen der Fallorganisationen vorgefunden wurde, war wertebasiert und sah die Führungskräfte in der Funktion der Unterstützenden oder Coaches. Die Aufgabe von Führungskräften in selbstorganisierten Teams war es, Orientierung zu geben und als 
Reflexionspartnerin/-partner zu agieren, Rahmenbedingungen für Partizipation und Selbstorganisation zu gestalten sowie Hindernisse abzubauen und bei der Konfliktbearbeitung zu unterstützen.

Die damit verbundenen neuen Rollenanforderungen erzeugten in den Fallorganisationen bei einigen Führungskräften Widerstand, da mit diesen Anforderungen für sie ein Kontroll- und Machtverlust einherging. Dieser Verlust wurde für die Führungskräfte zum Problem, wenn sie ihre gesamte Legitimation als Führungskraft in Gefahr sahen. Andere hingegen begrüßten zum Beispiel die Verbreitung einer „Speak-up-Mentalität“, weil sie ihre Beschäftigten als bessere Sachverständige schätzten und überzeugt waren, durch den partizipativen Diskurs zu besseren Lösungen zu kommen.

Die beschriebenen Widerstände waren in den Organisationen wenig(er) ausprägt, wo sich Strukturen kontinuierlich entwickelten, von Anfang an auf transparent kommunizierten Werten fußten und auch bei der Selektion von Führungskräften berücksichtigt wurden. Werte können dann als Antrieb zum Arbeiten dienen.

Die Unterstützungsrolle fiel den Führungskräften, deren persönliche Werte von vorne herein eine hohe Übereinstimmung mit den Organisationswerten aufwiesen, nicht schwer. Sie sahen ihre Herausforderung eher darin, die Beschäftigten zu befähigen, eigenständig Entscheidungen zu treffen:

"Sparringspartner zu SEIN, aber trotzdem in diesen Teams Spezialisten $z u$ haben, dass ein Teamlead auch nicht alles wissen muss, ALLES können muss, sondern es ist VIEL auf Vertrauensarbeit beziehungsweise zu sagen, okay, ihr dürft das entscheiden, macht das so, wie es für euch gut passt, wir vertrauen euch da. Aber sie natürlich dafür auch zu befähigen, ne? Also da haben auch ganz viele erstmal so: "Darf ich das machen? Ist das mein Bereich? Kann ich das, diese Entscheidung treffen?" Und da wollen wir eigentlich die Leute hinbringen: "Ja, ihr dürft das." (F3W 04HR: 110).

\section{Diskussion}

Die Prozesse in den Unternehmen, die die flexiblen, agilen Konzepte durchgängig implementiert hatten, waren insbesondere von starker Zielorientierung und -klarheit geprägt. In diesen Unternehmen wurde auf Basis umfassender Transparenz zum Beispiel durch den Einsatz entsprechender Managementsoftware ein hohes Maß an Partizipation und Flexibilität ermöglicht. Die Kommunikation war klar strukturiert und fand häufig, regelmäßig und im Rahmen standardisierter Formate statt (Passung von Kommunikationszweck und-medium). Sie umfasste unter anderem partizipative Prozesse der Zielentwicklung und -reflexion als Orientierungsrahmen. Eine vertrauensbasierte und unterstützende Haltung der Führungskräfte und Organisationsleitung spielte eine wichtige Rolle für die erfolgreiche Anwendung agiler Arbeitsweisen. Diese Ergebnisse werden im Folgenden in Hinblick auf ihre Bedeutung für das Wohlbefinden und die Gesundheit bei der Arbeit diskutiert. Dazu werden die dargestellten Ergebnisse zu den Kennzeichen agiler Organisationen in den aktuellen Forschungsstand zum Thema Wohlbefinden Gesundheit bei der Arbeit eingeordnet.

Als wichtige Voraussetzungen für die erfolgreiche Anwendung agiler Arbeitsweisen konnte ein hohes Maß an Transparenz in Hinblick auf fachliche Informationen, Ressourcenallokation, Bearbeitungsstände und wirtschaftliche Indikatoren identifiziert werden.
Dieser Befund ist im Einklang mit bisherigen Erkenntnissen zur Bedeutung von Transparenz für organisationalen Erfolg, Führungsund Teamerfolg (siehe z. B. Schnackenberg u. Tomlinson 2014; Tonkin 2013). Hier konnten die Vorteile einer zunehmenden Digitalisierung der Arbeit genutzt werden. Durch den Einsatz entsprechender digitaler Tools konnten nicht nur Informationen in umfassender Weise zugänglich gemacht werden, sondern auch die Möglichkeiten des zeit- und ortsflexiblen Arbeitens erleichtert werden.

Die damit verbesserte Vereinbarkeit von Arbeits- und Privatleben, die von den Befragten als positives Merkmal ihrer Arbeitsweise genannt wurde, stand in einer Reihe von Vorteilen, die von den Beschäftigten mit der Möglichkeit der selbstorganisierten Arbeit von zu Hause verbunden wurden. Zeit- und Ortsflexibilität geht auf Seiten der Beschäftigten häufig mit einer höheren Arbeitszufriedenheit, geringeren Pendelzeiten und -kosten sowie verbesserten Möglichkeiten des ungestörten Arbeitens von zu Hause einher. Dem gegenüber müssen aber auch Risiken der Flexibilitätsoptionen betrachtet werden. Die Arbeit von zu Hause führt häufig zu einer Auflösung der Grenzen zwischen Arbeits- und Privatleben (Kossek et al. 2006). Diese Entgrenzung bezieht sich etwa auf Kontaktanfragen aus dem Arbeitskontext außerhalb der Arbeitszeit. Beschäftigte, die von Zuhause arbeiten, berichten häufiger von sehr langen Arbeitszeiten und verkürzten Ruhezeiten (Wöhrmann et al. 2020).

Ein hohes Maß an Selbstorganisation kann insofern mit Arbeitsintensivierung und interessierter Selbstgefährdung einhergehen (Kubicek et al. 2017), die nicht nur gesundheitliche Folgen nach sich ziehen können, sondern auch die Balance zwischen Arbeit und Privatleben gefährden (Beermann et al. 2019). Für die gesundheitlichen Auswirkungen der Arbeit von zu Hause ist die Ausgestaltung von Prozessen und Arbeitsbedingungen entscheidend (BMAS 2020; Backhaus et al. 2020; Kunze et al. 2020).

Neben den Flexibilitätsmöglichkeiten zeigte sich ein wichtiges Merkmal agiler Organisationen in ausgeprägten Partizipationsmöglichkeiten und reflexiven Strukturen sowie Prozessen. Entsprechend ihrer Ausgestaltung bieten insbesondere reflexive Strukturen in einem volatilen Marktumfeld, das von beschränkter Planbarkeit geprägt ist, die Möglichkeit, Arbeitsbedingungen gesundheitsförderlich zu gestalten. Die beobachteten Prozesse der partizipativen Zielaushandlung und -anpassung lassen sich mit Moldaschl (2006) als institutionalisierte Reflexivität bezeichnen. Eine solche institutionalisierte Reflexivität bietet strukturelle Möglichkeiten zur diskursiven, kritischen Auseinandersetzung mit organisationalen Prozessen, Arbeitsweisen und Strukturen, um sich von Pfadabhängigkeiten zu distanzieren (Moldaschl 2006). Das Argument „Das haben wir schon immer so gemacht" verliert dadurch an Bedeutung. In unseren Fallstudien zeigten sich solche Strukturen beispielsweise in den eigens für die Aushandlung von Teamprozessen geschaffenen Räumlichkeiten oder der Bereitstellung technischer Softwarelösungen zur Herstellung von Transparenz und Partizipationsmöglichkeiten.

Die Nutzung reflexiver Strukturen setzt auf individueller Ebene eine Selbstauseinandersetzung voraus, die den Blick auch auf Belastungen und Ressourcen richtet und wichtiges Element für gesundheitliche Prävention ist. Reflexive Strukturen bieten die Möglichkeit, Belastungen zu erkennen, zu thematisieren und vor dem Hintergrund iterativer Prozesse entsprechende kurzfristige Anpassungen vorzunehmen. Einschränkend ist dabei aber zu berücksichtigen, dass 
Reflexivität in diesem Sinne auch personale Kompetenz erfordert, über die nicht alle Beschäftigten verfügen und die nicht ohne weiteres vorausgesetzt werden kann (Hallensleben et al. 2015; Wörlen 2020). Hier können Führungskräfte unterstützend tätig werden, was bei diesen ein entsprechendes Rollenverständnis voraussetzt.

Die Führung selbstorganisierter Teams erfordert in erster Linie eine Ausrichtung an den Teambedürfnissen (vgl. Abschnitt „Führungsverständnis“). Führungskräfte sollten die Zusammenarbeit erleichtern und Hindernisse beseitigen, die der Teamleistung im Wege stehen. Entsprechend der agilen Prinzipien sollten Führungskräfte eher einen Rahmen und Richtungen anstatt rigider Regeln vorgeben, um die Teams zu höherer Anpassungsfähigkeit zu befähigen (Parker et al. 2015). Ihre Aufgabe ist es, die Lernfähigkeit der Organisation und die Akzeptanz von Veränderungen zu fördern (Theobald et al. 2020). Dabei muss berücksichtigt werden, dass eine flexible, selbstorganisierte Arbeitsweise neue Herausforderungen der (digital vermittelten) zeit- und ortsflexiblen Zusammenarbeit mit sich bringt.

Treten bei vergleichsweise eigenständiger agiler Arbeit die Kontrolle und Weisung der Beschäftigten durch die Führungskraft in den Hintergrund, so rücken Vertrauens- und Beziehungsaufbau zwischen Führungskraft und den Teammitgliedern in den Vordergrund. Dies gilt jedoch als besonders schwierig, wenn der persönliche Kontakt ausbleibt oder reduziert wird (Liao 2017), wie es bei eigenständiger, zeitlich und örtlich verteilter Arbeit agiler Teams häufig der Fall ist. Eine besondere Anforderung für Führungskräfte ist daher, Vertrauen und Beziehung aufzubauen und zu pflegen, bei gleichzeitiger Unterstützung und Förderung der Eigenständigkeit und Flexibilität der Beschäftigten. Für Führungskräfte bedeutet dies, neben der Unterstützung bei den Arbeitsaufgaben insbesondere die individuellen Rahmenbedingungen und Bedürfnisse der Beschäftigten im Blick zu haben, zu berücksichtigen und dafür immer wieder den persönlichen Austausch zu suchen (Zaccaro u. Bader 2003). Die Entwicklung und Berücksichtigung von Werten und Normen können dabei einen Orientierungsrahmen geben (Cortelazzo et al. 2019; s. auch Abschnitt "Orientierung durch Werte und Ziele“).

Darüber hinaus ergeben sich bei vermehrter Nutzung digitaler Tools und Kommunikationsmittel weitere Anforderungen für Führung und Zusammenarbeit. Neben technischen Herausforderungen müssen beispielsweise die verschiedenen Kommunikationswege anlass- und bedarfsgerecht genutzt werden (Hoch 2019).

Das Vorhandensein und die Konsistenz von Orientierungsmarkern werden in der Managementliteratur seit Dekaden stark in ihrer Bedeutung betont und sollten eigentlich keine Ausnahme darstellen (Fugate 2012). Insbesondere die konkrete Umsetzung in Strategie und Ziele ist dabei besonders bedeutsam (Appelbaum et al. 2012). In der Praxis ist die Umsetzung einer starken Orientierung durch die Leitung und das Managementteam jedoch in der Regel eher schwach (Burnes 2012).

Zur Befähigung der Beschäftigten, selbstständig und verantwortungsvoll arbeiten zu können, bedarf es der Unterstützung beim Ausprobieren eigener Arbeitsweisen und bei der Reflexion von Lernerfahrungen (Amundsen u. Martinsen 2014). Dabei spielen Führungskräfte ebenfalls eine wichtige Rolle: Sie können im Rahmen ihrer Möglichkeiten der Gestaltung der Arbeitsbedingungen im Team beispielsweise Lerngelegenheiten bei der Arbeit schaffen, selbststän- diges Handeln anerkennen, zur Reflexion von Erfahrungen anregen und dabei unterstützen, sowie individuelle Lern- und Entwicklungsziele gemeinsam mit den Beschäftigten entwickeln (Ribbat 2020). Ein solches ermöglichendes Führungsverhalten geht in der Regel nicht nur mit Selbstwirksamkeit, Kreativität und Leistung der Teammitglieder einher, sondern ist auch mit größerer Arbeitszufriedenheit und Wohlbefinden verbunden (Cheong et al. 2019).

\section{Fazit}

Dieser Beitrag hat gezeigt, dass agile Arbeitsweisen eine Reihe von Chancen bieten, wie beispielsweise mehr Anpassungsfähigkeit, Flexibilität oder Autonomie. Es wurden einige Faktoren herausgearbeitet, die dabei unterstützen können, ihre Potenziale zu nutzen (z.B. Transparenz und Partizipation). Für die erfolgreiche und gesundheitsorientierte Umsetzung bedarf es auch bei agiler Arbeit geeigneter organisationaler Unterstützung für die Teams, die einzelnen Beschäftigten sowie die Führungskräfte. Agilität sollte kein Selbstzweck sein. Nutzen und potenzielle Risiken von flexibler und eigenverantwortlicher Arbeit sollten stets überprüft werden. Die Uneindeutigkeit des Agilitätsbegriffs bietet den Organisationen die Möglichkeit, entsprechend ihrer organisationsspezifischen Eigenlogik diesen für sich nutzbar zu machen. Entscheidend dabei ist zum einen, welche Voraussetzungen durch die oberste Führungsebene geschaffen werden und zum anderen, wie Teams und Führungskräfte die geschaffenen Freiräume auf Prozess- und Strukturebene gestalten. Führungsstile, die diesen Anforderungen gerecht werden, sind in im folgenden Beitrag von Emmerich und Rigotti vertiefend dargestellt und diskutiert.

Interessenkonflikt: Das Autorenteam gibt an, dass keine Interessenkonflikte vorliegen.

Ethikvotum: Die Ethikkommission der Bundesanstalt für Arbeitsschutz und Arbeitsmedizin hat sich am 09.08.2019 mit den ethischen Fragen des Forschungsvorhabens 027_2019_Weber: Fallstudien „Führung und Organisation im Wandel" (F2436) befasst. Der Antrag wurde insgesamt positiv bewertet.

Für alle Interviewteilnehmenden liegen Datenschutz- und Einwilligungserklärungen vor.

\section{Literatur}

Amundsen S, Martinsen ØL: Empowering leadership: Construct clarification, conceptualization, and validation of a new scale. The Leadership Quarterly 2014; 25: 487-511.

Appelbaum SH, Calla R, Desautels D, Hasan L: The challenges of organizational agility (part 1). Industrial and Commercial Training 2017; 49: 6-14.

Appelbaum SH, Habashy S, Malo J, Shafiq H: Back to the future: revisiting Kotter's 1996 change model". J Manage Develop 2012; 31: 764-782.

Backhaus N, Wöhrmann AN, Tisch A: BAuA-Arbeitszeitbefragung: Telearbeit in Deutschland. 2. Aufl. Dortmund: Bundesanstalt für Arbeitsschutz und Arbeitsmedizin, 2020.

Becke G: Agile Arbeitskonzepte zwischen Rationalisierung und gesundheitssensibler Gestaltung. In: Porschen-Hueck S, Jungtäubl M, Weihrich M (Hrsg.): Agilität? Herausforderungen neuer Konzepte der Selbstorganisation. Augsburg/München: Reiner Hampp Verlag, 2020.

Beermann B, Backhaus N, Tisch A, Brenscheidt F: Arbeitswissenschaftliche Erkenntnisse zu Arbeitszeit und gesundheitlichen Auswirkungen. Dortmund, 2019. 
Bell B, McAlpine KL, Hill SN: Leading from a distance: advancements in virtual leadership research. In: Landers RN (Hrsg.): The Cambridge Handbook of Technology and Employee Behavior. Cambridge University Press, 2019, S. 387-418.

Bendel O: Definition: Was ist "Agilität"? https://wirtschaftslexikon.gabler.de/ definition/agilitaet-99882/version-368852 (zuletzt aufgerufen, 02.07.2021)

BMAS (Bundesministerium für Arbeit und Soziales): Kurzexpertise - Verbreitung und Auswirkungen von mobiler Arbeit und Home-Office. Berlin, 2020.

Brüsemeister T: Qualitative Forschung. Ein Überblick. Wiesbaden: VS Verlag für Sozialwissenschaften, 2008.

Burnes B: Understanding the emergent approach to change. In: Boje M, Burnes B, Hassard J (Hrsg.): The Routledge Companion to Organizational Change. London: Routledge, 2012: 133-145.

Cheong M, Yammarino FJ, Dionne SD, Spain SM, Tsai CY: A review of the effectiveness of empowering leadership. The Leadership Quarterly 2019; 30: 34-58.

Döring N, Bortz J: Forschungsmethoden und Evaluation. Wiesbaden: Springer, 2016.

Dresing T, Pehl T: Praxisbuch Interview: Transkription \& Analyse. Anleitungen und Regelsysteme für qualitativ Forschende. 8. Aufl. Marburg: Audiotranskription, 2018.

Flick U, Kardorff E von, Steineke I: Qualitative Forschung: Ein Handbuch. 11. Aufl. Reinbek bei Hamburg: Rowohlt-Taschenbuch-Verlag, 2015.

Förster K, Wendler R: Theorien und Konzepte zu Agilität in Organisationen. Dresdner Beiträge zur Wirtschaftsinformatik 2012; 63. https://tud.qucosa.de/api/ qucosa\%3A27335/attachment/ATT-0/ (zuletzt aufgerufen 10.11.21).

Fugate M: The impact of leadership, management, and HRM on employee reactions to organizational change. In Martocchio JJ, Joshi A, Liao H (Hrsg.): Research in Personnel and Human Resources Management 2012: 177-208.

Haider SA, Theseen S, Khan S, Mata MN, Martins JM, Abreu A: A literature review on agility-is there a need to develop a new instrument? Int Journal of Entrepreneurship 2021; 25 (3).

Hallensleben T, Wörlen M, Moldaschl M: Institutional and personal reflexivity in processes of organisational learning. International Journal of Work Innovation 2015 1: 185-207.

Helfferich C: Die Qualität qualitativer Daten: Manual für die Durchführung qualitativer Interviews. Wiesbaden: VS Verlag für Sozialwissenschaften, 2011.

Hoch JE: Managing distributed work. Theorizing an IPO Framework. In: Landers RN (Hrsg.): The Cambridge Handbook of Technology and Employee Behavior. Cambridge University Press, 2019: 419-440.

Komus A, Kuberg M, Schmid S et al.: Studie Status Quo (Scaled) Agile 2019/2020. BPM-Labor für Business Process Management und Organizational Excellence. Hochschule Koblenz University of Applied Science 2020.

Kossek EE, Lautsch BA, Eaton SC: Telecommuting, control, and boundary management: Correlates of policy use and practice, job control, and work-family effectiveness. Journal of Vocational Behavior 2006; 68: 347-367.

Kubicek B, Paškvan M, Bunner J: The bright and dark sides of job autonomy. In: Korunka C, Kubicek B (Hrsg.): Job demands in a changing world of work: Impact on workers' health and performance and implications for research and practice. Berlin: Springer, S. 45-63.

Kunze F, Hampel K, Zimmermann S: Homeoffice in der Corona-Krise: eine nachhaltige Transformation der Arbeitswelt? Universität Konstanz, 2020.

Laanti M: Agile and wellbeing-Stress, empowerment, and performance in scrum and kanban teams. Proceedings of the 46th Hawaii International Conference on System Sciences 2013: 4761-4770.

Laloux F: Reinventing organizations: A guide to creating organizations inspired by the next stage in human consciousness. Nelson Parker, 2014.

Lamnek S, Krell C: Qualitative Sozialforschung. 6. Aufl. Weinheim: Beltz, 2016.

Liao C: Leadership in virtual teams: A multilevel perspective. Human Resource Management Review 2017; 27: 648-659.

Luhmann N: Vertrauen: Ein Mechanismus der Reduktion sozialer Komplexität. Stuttgart: Enke, 2000

Maturana HR, Varela J: Der Baum der Erkenntnis - Die biologischen Wurzeln menschlichen Erkennens. Bern/München: Scherz, 1987.

Meredith S, Francis D: Journey towards agility: the agile wheel explored. The TQM Magazine 2000

Moldaschl M: Innovationsfähigkeit, Zukunftsfähigkeit, Dynamic Capabilities: Moderne Fähigkeitsmystik und eine Alternative. In: Schreyögg G, Conrad P (Hrsg.): Managementforschung 16: Management von Kompetenz. Wiesbaden: Gabler, 2006, S. 1-36.
Mrugalska B, Ahmed J: Organizational agility in industry 4.0: A systematic literature review. Sustainability 2021; 13: 8272.

Nerdinger FW, Blickle G, Scharper N: Arbeits- und Organisationspsychologie. Berlin/Heidelberg: Springer, 2014.

Parker DW, Holesgrove M, Pathak R: Improving productivity with self-organised teams and agile leadership. International Journal of Productivity and Performance Management 2015.

Parsons T: The Social System. London: Routledge, 1951.

Parsons T: The social system. New Orleans: Quid Pro Books, 2012.

Porschen-Hueck S, Jungtäubl M, Weihrich M (Hrsg.): Agilität?: Herausforderungen neuer Konzepte der Selbstorganisation. Augsburg/München: Rainer Hampp Verlag, 2020.

Ribbat M: Führungskräfte als Gatekeeper für arbeitsintegriertes Lernen: Mit Learning Contracts die Gestaltung des digitalen Wandels ermöglichen. In: Richter G (Hrsg.): Lernen in der digitalen Transformation. Stuttgart: Schäffer Poeschel, 2020, S. 83-94.

Schermuly CC, Koch J: New Work und psychische Gesundheit. In: Badura B, Ducki A, Schröder H, Klose J, Meyer M (Hrsg.): Fehlzeiten-Report 2019. Digitalisierung gesundes Arbeiten ermöglichen. Berlin: Springer, 2019, S. 127-139.

Schnackenberg AK, Tomlinson EC: A new perspective on managing trust in organization-stakeholder relationships. Journal of Management 2016; 42: 1784-1810.

Strauss AL, Corbin J: Grounded Theory: Grundlagen Qualitativer Sozialforschung. Weinheim/Beltz: Psychologie Verlags Union, 1996.

Theobald S, Prenner N, Krieg A, Schneider K: Agile leadership and agile management on organizational level-a systematic literature review. In: International Conference on Product-Focused Software Process Improvement. Cham, 2020, S. 20-36.

Tonkin, TH: Authentic versus transformational leadership: Assessing their effectiveness on organiza-tional citizenship behaviorof followers. International Journal of Business and Public Administration 2013; 10: 40-61.

Tuomivaara S, Lindholm H, Känsälä M: Short-term physiological strain and recovery among employees working with agile and lean methods in software and embedded ICT systems. International Journal of Human-Computer Interaction 2017: 857-867.

Vázquez-Bustelo D, Avella L, Fernández E: Agility drivers, enablers and outcomes: Empirical test of an integrated agile manufacturing model. International Journal of Operations E Production Management 2007; 27: 1303-1332.

Venkatesh V, Thong J, Chan FKY, Hoehle H, Spohrer K: How agile software development methods reduce work exhaustion: Insights on role perceptions and organizational skills. Information Systems Journal 2020; 30: 733-761.

VERBI Software: MAXQDA 2020 [computer software]. Berlin, Germany: VERBI Software, 2020

Walter A-T: Organizational agility: ill-defined and somewhat confusing? A systematic literature review and conceptualization. Management Review Quarterly 2021; 71: 343-391.

Wöhrmann AM, Backhaus N, Tisch A, Michel A: BAuA-Arbeitszeitbefragung: Pendeln, Telearbeit, Dienstreisen, wechselnde und mobile Arbeitsorte. Dortmund, 2020.

Wörlen M: Reflexive Projektorganisation, gute Projektarbeit - Welche Relevanz hat Agilität? In: Porschen-Hueck S, Jungtäubl M, Weihrich M (Hrsg.): Agilität?: Herausforderungen neuer Konzepte der Selbstorganisation. Augsburg/München: Reiner Hampp Verlag, 2020.

Yin RK: Case Study Research and Applications: Design and Methods. 6. Aufl. Los Angeles: SAGE, 2018.

Yusuf YY, Sarhadi M, Gunasekaran A: Agile manufacturing: The drivers, concepts and attributes. International Journal of Production Economics 1999; 62: 33-43.

Zaccaro SJ, Bader P: E-leadership and the challenges of leading e-teams: Minimizing the bad and maximizing the good. Organizational Dynamics 2003; 31: 377-387.

\section{Kontakt}

\section{Dr. Corinna Weber}

Bundesanstalt für Arbeitsschutz

und Arbeitsmedizin (BAuA)

Fachgruppe Arbeitszeit und Organisation

Friedrich-Henkel-Weg 1-25

44149 Dortmund

weber.corinna@baua.bund.de 